\title{
Synergies of herbal teas on improved plasma antioxidant capacity and postprandial hypoglycemic response in healthy adults
}

\author{
Anoma Chandrasekara $^{\mathrm{a}^{*}}$, Apeksha Herath ${ }^{\mathrm{a}}$, Rupika Abeynayake ${ }^{\mathrm{b}}$, \\ Adriano Costa de Camargo ${ }^{c}$ and Fereidoon Shahidid
}

\begin{abstract}
aDepartment of Applied Nutrition, Wayamba University of Sri Lanka, Makandura, Gonawila, 60170, Sri Lanka
${ }^{b}$ Department of Agri Business Management, Wayamba University of Sri Lanka, Makandura, Gonawila, 60170, Sri Lanka

'Laboratory of Antioxidants, Nutrition and Food Technology Institute, University of Chile, Santiago, Chile

${ }^{\mathrm{d} D e p a r t m e n t}$ of Biochemistry, Memorial University of Newfoundland, St. John's, NL, A1B 3X9, Canada

*Corresponding author: Anoma Chandrasekara, Department of Applied Nutrition, Wayamba University of Sri Lanka, Makandura, Gonawila,60170, Sri Lanka. E-mail: anomac@wyb.ac.lk; anomapriyan@yahoo.ca

DOI: $10.31665 /$ JFB.2021.13262

Received: March 21, 2021; Revised received \& accepted: March 31, 2021

Citation: Chandrasekara, A., Herath, A., Abeynayake, R., de Camargo, A.C., and Shahidi, F. (2021). Synergies of herbal teas on improved plasma antioxidant capacity and postprandial hypoglycemic response in healthy adults. J. Food Bioact. 13: 82-92.
\end{abstract}

\begin{abstract}
Herbal teas play a major role in the global traditional food cultures. There are a variety of herbal teas used for general wellness and prevention and management of chronic diseases including diabetes mellitus. The present study aimed to determine the phenolic content and antioxidant activities of herbal teas prepared with matured jackfruit (Artocarpus heterophyllus) leaves after senescence and abscission, and dried Cassia (Cassia auriculata) flowers and buds. The sensory evaluation was performed using 50 untrained panelists to determine the amount of herbal ingredient to be used in the preparation of herbal tea. Furthermore, the potential single dose efficacy of three herbal teas, namely, Jackfruit leaves tea (JLT), Cassia flower tea (CFT) and mixed jackfruit leaves and Cassia flower tea (JCT) on postprandial glycemic response and plasma antioxidant capacity (PAC) of healthy adults were investigated. Phenolics were identified using high performance liquid chromatography-tandem mass spectrometry. A randomized crossover study was led with 16 healthy adults who consumed $250 \mathrm{~mL}$ of freshly prepared herbal beverage with $50 \mathrm{~g}$ glucose and $50 \mathrm{~g}$ glucose in $250 \mathrm{~mL}$ water as the control randomly within three visits with a washout period of four days between two visits. Blood samples were collected using microcapillary tubes at the baseline and postprandial at 30, 45, 60,90, and $120 \mathrm{~min}$. The plasma was analyzed for glucose concentration (PGC) and PAC. JLT and JCT showed a significant reduction of PGC compared to the control at 30,45 , and 60 min time points. JLT and JCT showed higher efficacy in plasma glucose reduction than CFT. All herbal teas significantly increased PAC at the end of 120 min post ingestion. Further research is warranted to examine the synergistic effect of two mixed ingredients for herbal tea and the long-term efficacy of multiple dose ingestion of respective herbal teas in the prevention and management of hyperglycemia and type 2 diabetes.
\end{abstract}

Keywords: Artocarpus heterophyllus; Cassia auriculata; HPLC; Mixed ingredients.

\section{Introduction}

Sedentary lifestyle pattern and unhealthy dietary choices play a critical role instigating obesity, postprandial hyperglycemia and hyperlipidemia that cause enhanced oxidative stress leading to inflammation and metabolic derangement. Diabetes mellitus (DM) is a group of metabolic disorders characterized by increased blood glucose levels and is at increase all over the world at an alarm- 
ing rate. According to the International Diabetic Federation (IDF, 2019), half a billion people live with DM worldwide. Furthermore, an increase of $51,33,74,55$ and $143 \%$ cases was estimated in the world, North America and Caribbean, South East Asia, South and Central America, and Africa, respectively, by the year 2045 (IDF, 2019). Persistent high blood glucose levels exert vascular damages that affecting heart, eyes, kidneys and nerves, and reduce the quality of life and increase healthcare cost and mortality of the people in affected countries.

For centuries herbs have been used for their preventive and medicinal values of diseases. Herbal teas are popular among other common beverages in their habitual food cultures in many countries (Chandrasekara and Shahidi, 2018). In addition to fulfilling part of the daily fluid requirement, herbal teas serve a number of purposes including boosting of optimum health, management of several diseases and reducing the risk of ailments with aging in addition to serving as cosmetics (Chandrasekara and Shahidi, 2018). The trend among individuals with diabetes to use complementary and alternative medicine (CAM) treatment modalities is increasing worldwide and include herbal teas, encompassed with other potential dietary supplements. The external sources of phenolic antioxidants are reasonably helpful in attenuating defied level of oxidative stress in the body due to overproduction of free radicals and reactive oxygen species (ROS). In addition, phenolic compounds can inhibit alpha-amylase and alpha-glucosidase, which are hydrolases related to the absorption of carbohydrates (Pinaffi et al., 2020; Li and Tsao, 2019; Silva et al., 2019).

In the recent past, popularity of herbal teas as natural health products has increased (Li et al., 2019). Several types of herbal teas are consumed by health-conscious consumers in different countries depending on the availability of the respective plants in their localities. Herbal teas are rich sources of a number of groups of bioactive compounds such as carotenoids, phenolic acids, flavonoids, coumarins, alkaloids, polyacetylenes, saponins and terpenoids. Antioxidant, antibacterial, antiviral, anti-inflammatory, antihyperglycemic, antihyperlipidemic, anticarcinogenic and antiaging activities are some of the expected health effects of these compounds (Shahidi, et al., 2019; Zhao et al., 2019; Akalin et al., 2019; Chandrasekara and Shahidi, 2018; Chandrasekara et al., 2018).

Cassia auriculata L (Ranawara), commonly known as Tanner's Cassia, is a plant which grows abundantly in India and Sri Lanka. It is widely used in Ayurvedic medicine as a tonic, astringent and as a remedy for diabetes, conjunctive and ophthalmic conditions. Dried flower and leaves of the plant are used for therapeutic purposes (Kalaivani and Umamaheswari, 2008). The extract of dried flowers of Cassia exhibited demonstrated potent antihyperglycemic activity against maltose challenge in a diabetic rat model, similar to the drug acarbose, and also showed strong alpha-glycosidase inhibitory activity (Abesundara et al., 2004).

Artocarpus heterophyllus (jackfruit) is a large evergreen tree belonging to the family Moraceae abundant in Asia including China, India, Malaysia, Bangladesh, Philippines, Indonesia and Sri Lanka and Africa and in some regions of South America (Wang et al., 2017). The parts of the tree have been used traditionally in folklore medicine to treat a number of ailments (Prakash et al., 2009). A number of flavones, namely morin, dihydromorin, cynomacurin, artocarpin, isoartocarpin, cylcoartocarpin, artocarpesin, oxydihydroartocarpesin, artocarpetin, norartocarpetin, cycloartinone and artocarpanone have been reported from different morphological parts of A. heterophyllus (Rama Rao et al., 1973). The leaves have been used in attenuating several disease conditions such as hyperglycemia, hyperlipidemia, antioxidant and antibacterial activities, among others (Thapa et al., 2016; Biworo et al., 2015; Swami et al., 2012; Omar et al., 2011; Loizzo et al., 2010; Chandrika et al.,
2006). Leaves are a rich source of phenolics, including tannins, and other bioactive constituents such as terpenoids, coumarin, alkaloids, chalcones, stibenoids, lignans, steroids and saponins (Thapa et al., 2016; Wang et al., 2017). In previous studies, fresh jack fruit leaves were used and reported several therapeutic effects in animal models (Chandrika et al., 2006).

In addition to using a single herb in tea preparation, a mix of two or more herbs are also used to improve sensory characteristics such as colour, taste and health effects, which may be influenced by their phenolic profiles (El-Azim et al., 2015; Tipduangta et al., 2019; de Camargo and Schwember, 2019). Furthermore natural product mixtures may provide effective health outcomes due to beneficial synergistic interactions among bioactive constituents therein (Caesar and Cech, 2019). However, there are no published reports on bioactivities of herbal tea preparations using a mix of matured jackfruit leaves after senescence and abscission and dried Cassia buds and flowers.

Furthermore, information on plasma antioxidant activities and postprandial glycemic response of herbal teas prepared with, matured jackfruit leaves and Cassia buds and flowers are limited. Therefore, the objective of the present study was to determine the phenolic content and antioxidant activities of herbal teas prepared with matured jackfruit leaves after senescence and abscission and dried Cassia buds and flowers. In addition, the single-dose efficacy of herbal teas on postprandial glycemic response and plasma antioxidant capacity of healthy adults were determined.

\section{Materials and methods}

\subsection{Herbal samples}

Dried buds and flowers of Tanner's Cassia (Cassia auriculata) were purchased from a local market in Sri Lanka and matured jackfruit leaves after senescence and abscission belonging to firm variety (Waraka) of jackfruit (Artocarpus heterophilus Lam.) were collected from home gardens in the North Western Province in Sri Lanka. Herbal parts were cleaned to remove debris and dust by washing and sun-dried before use.

\subsection{Chemicals}

Sodium nitrite and Folin-Ciocalteu's reagent were procured from Research Lab Fine Chem Industries (Mumbai, India). Sodium carbonate and ferric chloride were purchased from Thomas Baker (Chemicals) Ltd. (Mumbai, India). The compound 3-(2-pyridyl)-5,6diphenyl-1,2,4-triazine-4,4-disulfonic acid sodium salt (Ferrozine) was purchased from SERVA Electrophoresis GmbH, (Heildberg, Germany). The compounds 2,2-diphenyl-1-picrylhydrazyl (DPPH), 2,2'-azino-bis (3-ethylbenzothiazoline-6-sulfonate) (ABTS), trolox, ferric chloride, ferrous chloride, 2,2-azobis (2-methylpropionamidine) dihydrochloride (AAPH), potassium ferricyanide, sodium chloride, gallic acid, catechin, ascorbic acid and methanol, were bought from Sigma-Aldrich, (St Louis, MO, USA). Aluminum chloride, and dibasic potassium phosphate were procured from Techno Pharm Chem (Delhi, India). Sodium hydroxide $(\mathrm{NaOH})$, and monobasic potassium phosphate, were purchased from Loba Chem Pvt Ltd (Mumbai, India). Ethylenediaminetetraacetic acid trisodium salt $\left(\mathrm{Na}_{3}\right.$ EDTA) was purchased from Needham Market (Suffolk, UK). Ellagic acid, protocatechuic acid (+)-catechin, (-)-epicatechin were purchased from Sigma Aldrich Canada Ltd (Oakville, ON, Canada). Acetonitrile, and formic acid, were procured from Fisher 
Scientific Ltd (Ottawa, ON, Canada). Glucose GOD-PAP reagent was purchased from BIOLABO (Maizy, France).

\subsection{Preparation of herbal extracts}

The hot water extracts of each herbal material were prepared separately by boiling dried jackfruit leaves, and Cassia buds and flowers, in water $(1: 15, \mathrm{w} / \mathrm{v})$ at $100{ }^{\circ} \mathrm{C}$ for $20 \mathrm{~min}$. The residues were filtered using medium porosity filter papers and extracts were freeze dried at $-55^{\circ} \mathrm{C}$, and 0.012 mbar (Alpha $1-4 \mathrm{LD}$ plus CHRIST, Osterode am Harz, Germany). Lyophilized extracts were stored at $-80{ }^{\circ} \mathrm{C}$ for further analysis.

\subsection{Identification and quantification of phenolic compounds of herbal extracts}

\subsubsection{HPLC-DAD-ESI-MS ${ }^{n}$ analysis}

RP-HPLC analysis of phenolic composition of aqueous extracts was carried out using an Agilent 1100 HPLC system (Agilent Technologies, Palo Alto, CA, USA) equipped with a G1311A quaternary pump, a G1379A degasser and a G1329A ALS automatic sampler, a G1330B ALS Therm, a G1316A Colcom column compartment, a G1315B diode array detector (DAD) and a system controller linked to Chem Station Data handling system (Agilent Technologies, Palo Alto, CA, USA) (de Camargo et al., 2015). A SUPELCOSIL ${ }^{\mathrm{TM}}$ LC-18 column $(4.6 \times 250 \mathrm{~mm}, 5 \mu \mathrm{m}$; Merck, Darmstad, Germany) was used for separation of phenolic compounds. Formic acid (1\%) was used as eluent $\mathrm{A}$ and $1 \%$ formic acid in acetonitrile as eluent $\mathrm{B}$ in the binary mobile phase. Gradient elution was used as follows; 0 min, $100 \% \mathrm{~A} ; 5 \mathrm{~min}, 90 \% \mathrm{~A} ; 35$ $\min , 85 \% \mathrm{~A} ; 45 \mathrm{~min}, 60 \% \mathrm{~A} ; 45$ to $50 \mathrm{~min} 60 \% \mathrm{~A}$ was maintained; from 50 to 55 min eluent $\mathrm{A}$ was increased and $100 \% \mathrm{~A}$ at $55 \mathrm{~min}$. The flow rate was maintained at $0.5 \mathrm{~mL} / \mathrm{min}$ and the compounds were detected at $280 \mathrm{~nm}$. Samples were filtered through a 0.45 $\mu \mathrm{m}$ PTFE membrane syringe filter (Thermo Scientific, Rockwood, TN, USA) before injection.

Identification of phenolic compounds was conducted using their relative retention times (RT), and UV spectra and ESI-MS spectra and comparison with authentic compounds. HPLC-MS analysis was performed using the same HPLC analytical conditions explained above with an Agilent 1100 series capillary liquid chromatography/mass selective detector (LC/MSD) ion trap system in electrospray ionization (ESI) negative ion mode. Complete system control and data evaluation were performed using Agilent LC/MSD Trap software (Agilent Technologies, Palo Alto, CA, USA). The mass spectrometer was operated in scan range of $\mathrm{m} / \mathrm{z} 50-2,000$; smart parameter setting using a drying gas $\left(\mathrm{N}_{2}\right)$ temperature of 350 ${ }^{\circ} \mathrm{C}$, drying gas flow $12 \mathrm{~L} / \mathrm{min}$, and nebulizer gas $\left(\mathrm{N}_{2}\right)$ pressure of $70 \mathrm{psi}$. A number of compounds present in the extracts were tentatively identified using ESI-MS ${ }^{\mathrm{n}}$ and UV spectral data and those in the available literature and quantified as equivalents of the closely related standards. The external standard method in which reference compounds were chromatographed under similar conditions separately from samples was used for quantification purposes.

\subsubsection{Determination of total phenolic content (TPC) of herbal extracts}

The total phenolic contents of herbal extracts were determined ac- cording to the method of Singleton and Rossi (1965) as explained in Chandrasekara et al (2020). Briefly, a methanolic solution of herbal extract was prepared using the lyophilized crude extracts at a concentration of $1 \mathrm{mg} / \mathrm{mL}$. Folin Ciocalteu's reagent $(0.25 \mathrm{~mL})$ was added to centrifuge tubes containing $0.25 \mathrm{~mL}$ of each extract and the contents were mixed thoroughly by vortexing. To the reaction mixture $0.5 \mathrm{~mL}$ of saturated sodium carbonate was added, followed by the addition of distilled water $(4 \mathrm{~mL})$ to each tube and thorough mixing. Tubes were allowed to stand at room temperature in the dark for $35 \mathrm{~min}$ followed by centrifugation at 4,000 $\times \mathrm{g}$ for 10 min (Refrigerated centrifuge 3-18R TOMOS Life Science Group, Belmont, MA, USA). The absorbance of the resultant blue color supernatant was read at $725 \mathrm{~nm}$ (UV-VIS Spectrophotometer, Labomed Inc, Culver City, CA, USA) using appropriate blanks for background subtraction. The content of total phenolic in each extract was determined based on a standard curve of gallic acid and expressed as $\mu \mathrm{mol}$ gallic acid equivalents (GAE) per gram of crude extract.

\subsection{Determination of total flavonoid content (TFC)}

Total flavonoid content was determined using a colorimetric method as previously reported (Chandrasekara et al., 2020). In this a known volume $(1 \mathrm{~mL})$ of aliquot of the extract, dissolved in methanol $(1 \mathrm{mg} / \mathrm{mL})$, was mixed with $4 \mathrm{~mL}$ of distilled water in a $50 \mathrm{~mL}$ centrifuge tube and $0.3 \mathrm{~mL}$ of $5 \% \mathrm{NaNO}_{2}$ was then added to the tube and allowed to react for $5 \mathrm{~min}$. Subsequently, $0.3 \mathrm{~mL}$ of $10 \%$ $\mathrm{AlCl}_{3}$ was added to the reaction mixture and allowed to stand for 1 min. Finally, $2 \mathrm{~mL}$ of $1 \mathrm{M} \mathrm{NaOH}$ and $2.4 \mathrm{~mL}$ of distilled water were added and mixed immediately. Centrifuge tubes were kept in the dark at room temperature for $15 \mathrm{~min}$ followed by centrifugation at $4,000 \times g$ for $5 \mathrm{~min}$. The absorbance was read at $510 \mathrm{~nm}$ against a blank prepared in a similar manner by replacing the extract. Catechin was used to prepare the standard curve and the total flavonoid content was calculated and expressed as $\mu \mathrm{mol}$ catechin equivalents (CE) per gram of crude extract.

\subsection{DPPH radical scavenging activity (DRSA)}

The DPPH radical scavenging ability was determined by the method of Lee et al. (2007) as explained by Kumari et al. (2019). The sample of $0.04 \mathrm{~mL}$ of extract $(1 \mathrm{mg} / \mathrm{mL}$ in methanol) was added to the $1.96 \mathrm{ml}$ of methanolic DPPH $(60 \mu \mathrm{M})$ solution. After the mixture was vortexed, it was allowed to stand at room temperature in the dark for $20 \mathrm{~min}$. The absorbance of the solutions was red at $517 \mathrm{~nm}$ with appropriate blank. The DPPH radical scavenging activity was expressed as $\mu \mathrm{mol}$ trolox equivalents (TE) per gram of crude extract.

\subsection{Trolox equivalent antioxidant capacity (TEAC)}

The TEAC of extracts was determined according to the method previously explained by Chandrasekara and Shahidi (2010). AAPH $(2.5 \mathrm{mM})$ was mixed with ABTS $(100 \mathrm{mM})$ in saline phosphate buffer (PBS) $(\mathrm{pH} 7.4,0.15 \mathrm{M} \mathrm{NaCl})$ to prepare the $\mathrm{ABTS}^{\circ+}$ solution. The solution was kept in a water bath at $60^{\circ} \mathrm{C}$ for $16 \mathrm{~min}$ and the flask was covered with an aluminum foil to protect it from light. Medium porosity filter papers were used to filter the prepared $\mathrm{ABTS}^{-+}$solution before mixing with the extract. A separate blank was used to reduce the diminished absorbance of radical solution. PBS solution was used to prepare herbal extract $(1 \mathrm{mg} / \mathrm{mL})$ 
which was further diluted to fit within the range of $6.25-50 \mu \mathrm{M}$ of the standard curve prepared using trolox. To measure the TEAC, $40 \mu \mathrm{L}$ of the extract were mixed with $1,960 \mu \mathrm{L}$ of the ABTS ${ }^{*}$ solution. The absorbance of reaction mixture was measured at $734 \mathrm{~nm}$ immediately at the point of mixing $\left(\mathrm{t}_{0}\right)$ and after $6 \min \left(\mathrm{t}_{6}\right)$. The decrease in th absorbance at $734 \mathrm{~nm}$ after $6 \mathrm{~min}$ of addition of trolox and extract was calculated using the equation: $\Delta \mathrm{A}$ trolox $=\left(\mathrm{At}_{0}\right.$, trolox $-\mathrm{At}_{6}$, trolox $)-\left(\mathrm{At}_{0}\right.$, blank- $\mathrm{At}_{6}$, blank $)$, where $\Delta \mathrm{A}$ is the reduction of absorbance and $\mathrm{A}$ is the absorbance at a given time. TEAC values were expressed as $\mu \mathrm{mol}$ trolox equivalents (TE)/g of crude extract.

\subsection{Reducing power (RP)}

The determination of RP of samples was carried out using a spectrophotometric method as explained by Chandrasekara et al. (2020). Briefly, $0.5 \mathrm{~mL}$ of extract was mixed with $1.25 \mathrm{~mL}$ of phosphate buffer solution $(0.2 \mathrm{M}, \mathrm{pH} 6.6)$ and $1.25 \mathrm{~mL}$ of potassium ferricyanide in a centrifuge tube. The mixture was incubated for $20 \mathrm{~min}$ at $50{ }^{\circ} \mathrm{C}$ and $1.25 \mathrm{~mL}$ of $10 \%$ TCA were added followed by centrifugation at $1,750 \times g$ for $10 \mathrm{~min}$. The supernatant $(1 \mathrm{~mL})$ was transferred into a tube containing $1.25 \mathrm{~mL}$ of deionized water and $0.25 \mathrm{~mL}$ of $0.1 \%(\mathrm{w} / \mathrm{v}) \mathrm{FeCl}_{3}$, and the absorbance values were read using a spectrophotometer at $700 \mathrm{~nm}$. The absorbance values were read at $700 \mathrm{~nm}$. The results were expressed as $\mu \mathrm{mol}$ ascorbic acid equivalents (AAE) per gram of crude extract using a standard curve prepared using ascorbic acid.

\subsection{Ferrous ion chelating activity (FICA)}

The ability of herbal tea extracts to chelate ferrous ions was measured as explained by Chandrasekara et al. (2020). Briefly, $0.4 \mathrm{~mL}$ of crude extracts in distilled water (1mg.mL) was added to a solution of $2 \mathrm{mM} \mathrm{FeCl}_{2}(0.05 \mathrm{~mL})$ followed by addition of $5 \mathrm{mM}$ ferrozine $(0.2 \mathrm{~mL})$. The total volume was adjusted to $4 \mathrm{~mL}$ with distilled water followed by the vigorous vortexing. After leaving $10 \mathrm{~min}$ at room temperature the absorbance of the reaction mixture was measured at $562 \mathrm{~nm}$. Distilled water was used as the control. Appropriate blanks were prepared with $0.4 \mathrm{~mL}$ of the sample and $3.6 \mathrm{~mL}$ of distilled water for background subtraction. The inhibition percentage of ferrozine-ferrous ion complex formation was calculated by the following equation. Metal chelating effect $(\%)=$ $\{1-($ Absorbance of the sample/Absorbance of the control $)\} \times 100$. The percent inhibition of ferrozine-ferrous ion complex formation was calculated by the following equation: metal chelating effect $(\%)=[1-($ absorbance of the sample - absorbance of the control $)]$ 100 . The results were expressed as $\mu \mathrm{mol}$ EDTA equivalents per gram of crude extract.

\subsection{Testing of herbal tea preparation}

Preliminary sensory studies were conducted to determine the level of herbal ingredient for tea preparation based on color, taste preference, acceptance, and willingness of panel members to drink herbal tea regularly. Three types of herbal teas, each with three formulations were prepared. Three formulations of Cassia flower tea (CFT) were prepared with dried Cassia flowers and buds in water $(1: 500,1: 200$, and 1:100; w/v). Three formulations of jackfruit leaves tea (JLT) included matured jackfruit leaves in water in the ratio of 1:100, 1:50 and 1:25 (w/v). Jackfruit leaves and Cassia flower mixed tea (JCT) three formulations were prepared using dried Cassia flowers and buds and jackfruit leaves in the ratio of $1: 13,1: 6$ and 1:8 $(\mathrm{w} / \mathrm{w})$ and ingredients in water in the ratio of $1: 24,1: 48$ and $1: 37(\mathrm{w} / \mathrm{v})$, respectively. Each tea was prepared by adding ingredient(s) to $1 \mathrm{~L}$ of water and then allowed to boil for 20 min with medium heat followed by 20 min standing at room temperature. Residues were discarded by straining and the lukewarm tea was used.

\subsection{Sensory evaluation of herbal teas}

Sensory properties were evaluated to select one formulation among three preparations for each herbal tea type using fifty untrained panelists ( 25 males and 25 females in the age range of 24-40 years old). The panelists were mainly students $(80 \%)$ and university staff $(20 \%)$ of the Makandura premises of Wayamba University of Sri Lanka. For each type of tea, three formulations, $50 \mathrm{~mL}$ each and coded with three digits, were served randomly to each panelist. Teas were served at the temperature range of $40-50{ }^{\circ} \mathrm{C}$ in transparent glass cups. The panelists were instructed to rinse their mouth with warm water before the commencement of tasting and in between each tasting of the formulation of herbal teas. The panelists waited 90 seconds before tasting the next sample and crackers were provided between tastings to eliminate any carry over effect. The panelists were not required to drink $50 \mathrm{~mL}$ of each tea and were asked to keep one sip of tea for 5 seconds in the mouth and swallow in small quantities. The panelists repeated tasting of the same tea whenever necessary.

The panelists used a 7-point hedonic scale for scoring five attributes, namely color, taste preference, after taste, overall acceptance, and willingness to drink regularly of the each formulation of the herbal tea. In the hedonic scale 1 meant 'dislike very much' and 7 meant 'like very much'. The herbal tea performed with high score with majority of attributes favorably was selected for further experimentation based on the results of Friedman ranking test (Milton, 1937).

\subsection{Determination of phenolic content of herbal teas}

The total phenolic contents of selected formulation of each herbal tea were determined as explained by Chandrasekara et al. (2018) using $0.25 \mathrm{~mL}$ of herbal tea with necessary dilution. The TPC in each herbal tea was expressed as $\mu \mathrm{mol}$ GAE per $250 \mathrm{~mL}$ serving.

\subsection{Determination of antioxidant activity of herbal teas}

Antioxidant activities of selected formulation of each herbal tea were determined by TEAC as explained by Chandrasekara and Shahidi (2010). The TEAC was expressed as $\mu$ mol TE per 250 mL serving.

\subsection{Determination of glycemic response and plasma antioxi- dant capacity}

\subsubsection{Subjects}

Sixteen healthy individuals with fasting blood glucose between 70 and $110 \mathrm{mg} / \mathrm{d}$ were recruited to the study after screening through an opened advertisement from Makandura premises of Wayamba University of Sri Lanka. The protocol was explained before their recruitment for the study and a written consent was obtained from 
each subject. Ethical approval was obtained from the Ethics Review Committee of Faculty of Livestock, Fisheries and Nutrition, Wayamba University of Sri Lanka (201810HI06). Those having any microvascular complications, alcohol and cigarette consumption, known allergies to herbal teas, and continuous use of any kind of medications were excluded from the study.

\subsubsection{Protocol}

A randomized single blind cross over study design was employed. Participants were asked to restrict their intake of tea, including any herbal teas and involvement in intensive physical activities such as exercises and running two days prior to a test day. Each person made four visits after 10-12 hours of fasting each day to complete the protocol and during the first visit anthropometric measurements (weight and height) and $24 \mathrm{~h}$ dietary recall were taken. On each study visit, a blood sample was collected immediately before administering herbal teas or control. Three test herbal teas, namely CFT, JLT and JCT, each $250 \mathrm{~mL}$ of serving with $50 \mathrm{~g}$ glucose and the control, $250 \mathrm{~mL}$ water with $50 \mathrm{~g}$ of glucose were served to participants in amber glass tumblers to eliminate the effect of color of teas and instructed to drink within $10 \mathrm{~min}$.

Blood samples were withdrawn by finger pricking at 30,45,60,90 and 120 min after completing the drink using glass micro-hematocrit capillary tubes ( $75 \mu \mathrm{L}$, sodium heparinized) for the determination of postprandial plasma glucose concentration (PGC). Only fasting and end point blood samples were taken by finger pricking for the determination of plasma antioxidant capacity (PAC). A four-day washout period was maintained between two test herbal teas. The plasma was separated using a micro-hematocrit centrifuge (Model HC-12A, Zenith Lab Inc, CA, USA) at 15,300 $\times g$ for $3 \mathrm{~min}$. The plasma samples were stored in Eppendorf tubes at -80 ${ }^{\circ} \mathrm{C}$ for subsequent analysis of PGC and PAC after completing the intervention study.

\subsubsection{Determination of plasma glucose concentration}

A commercial kit (Glucose GOD-PAP liquid ready for use from BIOLABO (Maizy, France) was used to determine the plasma glucose concentrations as explained by Chandrasekara et al (2020). The absorbance of the mixture of $10 \mu \mathrm{L}$ of plasma and $1 \mathrm{~mL}$ of reagent was read after $20 \mathrm{~min}$ at $30^{\circ} \mathrm{C}$ at $500 \mathrm{~nm}$ against the reagent blank. Plasma glucose values were expressed as $\mathrm{mg} / \mathrm{dL}$.

\subsubsection{Determination of plasma antioxidant capacity}

The TEAC assay described by Re et al. (1999) was used with minor modifications as explained by Kumari et al. (2020). Briefly, $20 \mu \mathrm{L}$ of the plasma sample was mixed with $980 \mu \mathrm{L}$ of the ABTS ${ }^{*}$ solution. The absorbance of the reaction mixture was read at 734 $\mathrm{nm}$ immediately at the point of mixing $\left(\mathrm{t}_{0}\right)$ and after $6 \mathrm{~min}\left(\mathrm{t}_{6}\right)$. The decrease in absorbance at $734 \mathrm{~nm}$ after $6 \mathrm{~min}$ of addition of trolox and plasma was calculated using the following equation: $\Delta \mathrm{A}$ trolox $=\left(\mathrm{At}_{0}\right.$, trolox $-\mathrm{At}_{6}$, trolox $)-\left(\mathrm{At}_{0}\right.$, blank $-\mathrm{At}_{6}$, blank $)$, where $\Delta \mathrm{A}$ is the reduction of absorbance and $\mathrm{A}$ is the absorbance at a given time. TEAC values were expressed as $\mu \mathrm{mol}$ TE per L.

\subsubsection{Statistical analysis}

All experiments regarding chemical analysis of test herbal extracts were carried out in triplicates and the data were reported as mean \pm standard deviation. The differences of mean values between two herbal extracts were determined by Student's $t$-test at $\mathrm{p} \leq 0.05$. Friedman analysis was performed to determine the mean ranking of sensory scores (Milton, 1937). The data of the glycemic response efficacy study were reported as mean difference \pm standard error of mean. Two factor repeated measures design was laid out for the study and General Linear Model with nested repeated measurers ANOVA was carried out to assess the plasma glucose concentration changes over time among the groups administered three herbal teas. The control and each herbal tea were compared using Bonferroni multiple comparison with $95 \%$ confidence interval (CI). The differences of group means at each time point were analyzed by paired $t$-tests with $95 \%$ CI. Plasma antioxidant capacities at the baseline and end of each herbal tea group were compared using paired $t$-tests. All statistical analyses were performed by hsing SPSS version 23 (IBM Analytics, USA).

\section{Results and discussion}

This study comprised of two phases. In the first phase, phenolic contents and antioxidant activities of two herbal extracts were determined using different in vitro methods. The identification of phenolic compounds of the extracts was performed by HPLCDAD-ESI-MS ${ }^{\mathrm{n}}$ analysis. In the second phase three selected herbal tea preparations based on sensory characteristics were tested in a randomized single blind cross over design to determine single dose efficacy on glycemic response and plasma antioxidant capacity of healthy adults. To the best of our knowledge, this study for the first time demonstrated single dose efficacy of herbal teas prepared with matured jackfruit leaves, Cassia buds and flowers and mixture on plasma antioxidant capacity and improved postprandial glycemic response of teas as potential dietary supplements in attenuating hyperglycemic conditions.

\subsection{Identification of phenolic compounds}

Individual phenolic compounds and their contents in aqueous extracts of dried Cassia flowers and buds, and jackfruit leaves are reported in Table 1. Several compounds belonging to different phenolic groups, such as phenolic acids, flavonoids, and proanthocyanidins were present.

The phenolic acids that were positively identified in Cassia flowers and jackfruit leaves included ellagic acid using an authentic standard and ESI-MS spectra. The flavonoids tentatively identified in Cassia flowers included gallocatechin, and derivatives of isorhamnetin, myricetin, and phloridzin (Table 1). Dimeric gallocatechin and trimeric propelargonidin were two major proanthocyanidins tentatively identified in aqueous extracts of Cassia flowers and their contents were 1,450 and $367 \mu \mathrm{g} / 100 \mathrm{~g}$ of crude extract, respectively. The results showed that compounds belonging to flavonoids were predominant in the aqueous extracts of Cassia flowers compared to phenolic acids (Table 1). In agreement, several flavonoids, namely kaempferol-3-O-rutiniside, kaempferol, quercetin and luteolin were identified from the arial parts of Cassia auriculata (Juan-Badaturugae et al., 2011).

In the present work ellagic acid and a derivative of monomeric flavonol, epigallocatechin, were identified in the aqueous extracts of jackfruit leaves and their contents were 0.17 and $436.4 \mu \mathrm{g} / 100$ $\mathrm{g}$ of extract, respectively (Table 1). A number of phytochemicals found in jackfruit leaves extracted with organic solvents and hot 
Table 1. Phenolic compounds identified and their contents $(\mu \mathrm{g} / 100 \mathrm{~g})$ in test herbal extracts

\begin{tabular}{lllll}
\hline Compound & {$[\mathbf{M}-\mathbf{H}]^{-}(\mathbf{m} / \mathbf{z})$} & Other product ions $(\mathbf{m} / \mathbf{z})$ & Cassia flowers and buds & Jackfruit leaves \\
\hline Ellagic acid & 301 & 283 & $1.23 \pm 0.06$ & $0.17 \pm 0.01$ \\
Gallocatechin* & 305 & 289 & $272.6 \pm 21.2$ & $\mathrm{nd}$ \\
Isorhamnetin derivative & 461 & 317 & $3726 \pm 52.2$ & $\mathrm{nd}$ \\
Myricetin derivative & 509 & 317 & $130.6 \pm 8.75$ & $\mathrm{nd}$ \\
Phloridzin derivative & 529 & 435 & $982.4 \pm 36.9$ & $\mathrm{nd}$ \\
Epigallocatechin derivative* & 579 & 305,179 & $\mathrm{nd}$ & $436.4 \pm 8.00$ \\
Dimeric gallocatechin* & 609 & 305 & $1,450 \pm 90.8$ & $\mathrm{nd}$ \\
Trimeric Propelargonidin* & 849 & $561,407,289$ & $367.0 \pm 0.80$ & $\mathrm{nd}$ \\
\hline
\end{tabular}

Data represent mean values for each sample \pm standard deviations $(n=3)$. Means followed by the same letter within a column are not significantly different $(p>0.05)$. $Q$ uantified as equivalent of catechin. nd: not detected.

water have previously been reported (Chandrika et al., 2006; Omar et al., 2011; El-Azim et al., 2015; Wang et al., 2017). Omar et al. (2011) isolated a flavonoid, isoquercitrin, from n-butanol extract of jackfruit leaves. El-Azim et al. (2015) reported identification and quantification of several phenolic acids, including ellagic acid and a number of flavonoids. However, in the present work we identified only two compounds, and this could be due to the use of hot water extracts of matured leaves after the senescence and abscission in the present work whereas other studies used dried powder of mature fresh leaves for the extraction of compounds. There could change the composition as well as the contents of phenolic compounds during senescence of leaves of jackfruit leaves. Kandil et al. (2004) showed changes of polyphenolic compounds of Rhizophora mangle leaves during development and senescence and reported that prior to leaf abscission flavonol glycosides and low oligomeric tannins disappeared from leaves leaving behind only large tannin polymers.

\subsection{Total phenolic contents of herbal extracts}

Table 2 presents total phenolic contents and antioxidant activities of aqueous herbal extracts used in the present study. The TPC of extracts were determined using Folin Ciocalteu's reagent. The TPC of two herbal extracts were 68.8 to $549.6 \mu \mathrm{mol} \mathrm{GAE} / \mathrm{g}$ of jackfruit leaves and Cassia flowers and buds extracts, respectively. All tested herbal materials were subjected to similar boiling conditions and solid to water ratio $(1: 15, \mathrm{w} / \mathrm{v})$ for the extraction. The extract of Cassia flowers had the highest TPC which was 8 times higher than that of jackfruit leaves.
Flavonoids have demonstrated antioxidant, anticancer, antiallergic, anti-inflammatory, and anti-neuro-inflammatory properties, among others (Shahidi et al., 2019; de Camargo et al., 2019; Zhang and Tsao, 2016). The TFC of crude extracts in the present study ranged from 8.3 to $102 \mu \mathrm{mol} \mathrm{CE} / \mathrm{g}$ extract (Table 2). The crude extract of Cassia flowers showed the highest TFC and was 13 times higher than that of jackfruit leaves and further showed that aqueous extracts of Cassia flowers were rich sources of phenolic compounds.

\subsection{Antioxidant activities of herbal extracts}

Table 2 presents the antioxidant activities of the tested aqueous herbal extracts. Except for FICA, Cassia flower extracts showed significantly $(p<0.05)$ higher level of TEAC, DRSA and RP compared to those of jackfruit leaves. The TEAC of the extracts of Cassia flowers were 22 times higher than that of Jackfruit leaves and were 523.9 and $23.4 \mu \mathrm{mol}$ trolox equiv/g of extract, respectively.

In DRSA assay antioxidant compounds donate a hydrogen atom or an electron to the stable DPPH radical to convert it to the nonradical form (Yeo and Shahidi, 2019). DRSA of Cassia flower extracts was 21 times higher than those of jackfruit leaves.

Compounds with reducing ability donate electrons to render their antioxidant effect. The RP of aqueous extracts of Cassia flowers and buds and Jackfruit leaves were 694 and $143 \mu \mathrm{mol}$ ascorbic acid equiv/g extract thus exhibited a considerable RP thereby acting as effective reductones. It was noteworthy that extracts of Cassia leaves had significantly higher RP than that of Jackfruit leaves which had 8 and 12 times more TPC and TFC, respectively.

Table 2. Phenolic contents and antioxidant activities (per $\mathrm{g}$ ) of extract of herbal teas

\begin{tabular}{lll}
\hline & Cassia flowers and buds & Senescent jackfruit leaves \\
\hline TPC ( $\mu$ mol gallic acid equiv) & $549.6 \pm 4.7^{\mathrm{a}}$ & $68.8 \pm 4.3^{\mathrm{b}}$ \\
TFC ( $\mu$ mol catechin equiv) & $102.2 \pm 2.3^{\mathrm{a}}$ & $8.3 \pm 2.4^{\mathrm{b}}$ \\
TEAC ( $\mu$ mol trolox equiv) & $523.9 \pm 12.0^{\mathrm{a}}$ & $23.4 \pm 6.6^{\mathrm{b}}$ \\
DRSA ( $\mu$ mol trolox equiv) & $567.7 \pm 7.9^{\mathrm{a}}$ & $27.1 \pm 2.8^{\mathrm{b}}$ \\
RP ( $\mu$ mol ascorbic acid equiv) & $693.8 \pm 5.7^{\mathrm{a}}$ & $143.0 \pm 5.1^{\mathrm{b}}$ \\
FICA ( $\mu$ mol EDTA equiv) & $0.03 \pm 0.01^{\mathrm{b}}$ & $0.09 \pm 0.01^{\mathrm{a}}$ \\
\hline
\end{tabular}

Abbreviations: Total phenolic content, TPC; Total flavanoid content, TFC; Trolox equivalent antioxidant capacity, TEAC; 2,2-diphenyl-1-picrylhydrazyl (DPPH) radical scavenging ability, DRSA; Reducing power, RP; Ferrous ion chelating ability, FICA; and Ethylenediaminetetraacetic acid, EDTA Values with the same letters in each raw for tested herbal extracts are not significantly different $(p>0.05)$. 
Table 3. Mean scores for sensory attributes and Friedman test ranks for different preparations of herbal teas

\begin{tabular}{|c|c|c|c|}
\hline \multicolumn{4}{|c|}{ Cassia flowers and buds tea (CFT) } \\
\hline & 459 & 353 & 721 \\
\hline Colour & $5.7 \pm 1.1^{\mathrm{a}}$ & $4.4 \pm 1.4^{b}$ & $4.4 \pm 1.4^{b}$ \\
\hline Taste preference & $5.9 \pm 1.1^{\mathrm{a}}$ & $4.7 \pm 1.3^{b}$ & $3.8 \pm 1.2^{c}$ \\
\hline After taste & $5.5 \pm 1.0^{a}$ & $4.7 \pm 1.1^{\mathrm{a}}$ & $3.6 \pm 1.1^{b}$ \\
\hline Overall acceptance & $5.3 \pm 1.1^{\mathrm{a}}$ & $5.3 \pm 1.2^{a}$ & $3.3 \pm 1.3^{b}$ \\
\hline Willingness to drink regularly & $5.2 \pm 1.3^{a}$ & $5.2 \pm 1.3^{a}$ & $3.5 \pm 1.1^{b}$ \\
\hline Friedman test mean rank & 2.43 & 2.06 & 1.51 \\
\hline \multicolumn{4}{|c|}{ Jackfruit leaves tea (JLT) } \\
\hline & 824 & 276 & 599 \\
\hline Colour & $5.8 \pm 1.5^{a}$ & $4.7 \pm 1.1^{b}$ & $4.8 \pm 1.3^{b}$ \\
\hline Taste preference & $5.4 \pm 1.1^{\mathrm{a}}$ & $3.7 \pm 1.3^{b}$ & $4.8 \pm 1.0^{c}$ \\
\hline After taste & $5.6 \pm 0.9^{a}$ & $4.4 \pm 1.1^{b}$ & $5.3 \pm 0.9^{a b}$ \\
\hline Overall acceptance & $5.2 \pm 1.2^{a}$ & $4.9 \pm 1.4^{a}$ & $5.0 \pm 1.4^{a}$ \\
\hline Willingness to drink regularly & $5.3 \pm 1.1^{\mathrm{a}}$ & $4.3 \pm 1.0^{b}$ & $4.8 \pm 1.3^{\mathrm{ac}}$ \\
\hline Friedman test mean rank & 2.26 & 1.87 & 1.87 \\
\hline \multicolumn{4}{|c|}{ Jackfruit leaves \& Cassia flowers mixed tea (JCT) } \\
\hline & 602 & 411 & 905 \\
\hline Colour & $6.1 \pm 1.5^{a}$ & $5.2 \pm 1.1^{b}$ & $5.5 \pm 1.3^{b}$ \\
\hline Taste preference & $5.9 \pm 1.2^{a}$ & $4.6 \pm 1.3^{b}$ & $3.7 \pm 1.0^{c}$ \\
\hline After taste & $5.4 \pm 1.2^{\mathrm{a}}$ & $4.4 \pm 1.3^{b}$ & $3.5 \pm 1.4^{c}$ \\
\hline Overall acceptance & $5.6 \pm 1.1^{a}$ & $5.4 \pm 1.3^{a}$ & $3.8 \pm 1.4^{b}$ \\
\hline Willingness to drink regularly & $5.5 \pm 1.1^{a}$ & $5.2 \pm 1.0^{\mathrm{a}}$ & $4.3 \pm 1.1^{c}$ \\
\hline Friedman test mean rank & 2.45 & 1.95 & 1.60 \\
\hline
\end{tabular}

All values are mean $\pm S D ; n=50$; Values in each row having the same letter are not significantly different $(p>0.05)$.

Furthermore, the compositional analysis showed that the aqueous extracts of Cassia flowers contained a high level of phenolic compounds belonging to phenolic acids, flavonoids and proanthocyanidins (Table 1). This explains the high antioxidant activity demonstrated by the extracts of Cassia flowers over Jackfruit leaves.

FICA of Cassia flowers extracts showed a lower value while those of jackfruit leaves exhibited 3 times higher value of 0.09 $\mu \mathrm{mol}$ EDTA equiv/g extract in this study. Compounds that act as chelating agents, reduce the concentration of metal ions available for catalyzing peroxidation thus serve as effective secondary antioxidants. In the body hydroxyl radicals are formed by ferrous ions via the Fenton's reaction. Presence of chelating agents decreases the intensity of the purple color of the complex of ferrous ions with Ferrozine. The reducing power of food phenolics is well recognized in many studies. Andjelković et al. (2006) determined the binding constants of selected phenolic acids and reported no complex formation for compounds lacking a catechol or galloyl moiety. Many compounds bearing these characteristics were identified in the herbal extracts evaluated in the present study. The ratio of ferric to ferrous ion is relevant for quick reaction during the initiation of lipid peroxidation via Fenton reaction.

Extracts of Cassia flowers showed the highest free radical scav- enging activity and reducing power between the herbal extracts tested in the present work. These activities correspond with the highest content of phenolics as determined by TPC and TFC determinations in the present work.

\subsection{Sensory evaluation}

In the present study, Cassia flower tea (CFT), jackfruit leaves tea (JLT) and mixed jackfruit leaves and Cassia flower tea (JCT) were prepared. Three formulations of each herbal tea were prepared and tested for evaluation of their sensory characteristics using a 7-scale hedonic scale. Table 3 presents the results of sensory evaluation of the tested herbal teas. The formulations, 459 of CFT, 824 of JLT and 602 of JCT received the highest mean rank according to the Frideman test for each herbal tea.

\subsection{Phenolic content and antioxidant (AO) activity of herbal tea preparations}

Total phenolic content and antioxidant activities of herbal tea prep- 
Table 4. Total phenolic content and antioxidant activity (per $250 \mathrm{~mL}$ of serving) of test herbal teas

\begin{tabular}{|c|c|c|}
\hline & $\begin{array}{l}\text { Total phenolic content } \\
\text { ( } \mu \text { mol gallic acid equiv) }\end{array}$ & $\begin{array}{l}\text { Trolox equivalent antioxidant } \\
\text { capacity ( } \mu \mathrm{mol} \text { trolox equiv) }\end{array}$ \\
\hline Cassia flowers tea (CFT) & $1843.9 \pm 16.4^{a}$ & $1880.8 \pm 40.5^{a}$ \\
\hline Jackfruit leaves + Cassia flowers tea (JCT) & $1722.0 \pm 14.8^{b}$ & $421.6 \pm 26.8^{b}$ \\
\hline Jackfruit leaves tea (JLT) & $670.1 \pm 3.1^{c}$ & $49.7 \pm 2.5^{c}$ \\
\hline
\end{tabular}

Values in each column having the same letters are not significantly different $(p>0.05)$

arations per serving of $250 \mathrm{~mL}$ are presented in Table 4 . The TPC ranged from 670 to $1,844 \mu$ mol GAE per serving and CFT had the highest content whereas JLT showed the least. The TPC of CFT, and JCT were 2.8 and 2.6 times higher $(p<0.05)$ than those of JLT, respectively. The antioxidant activity as assessed by TEAC of herbal teas ranged from 49.7 to $1,880.8 \mu \mathrm{mol}$ TE per serving of $250 \mathrm{~mL}$. Further, they followed the same order as that of TPC of herbal teas as CFT $>$ JCT $>$ JLT. It was noted that TEAC of CFT, and JCT were 37.6 , and 8.5 times higher $(p<0.05)$ than those of JLT, respectively. In agreement with the composition results obtained for the extracts of herbal materials in the present study (Table 2), CFT and JCT which were a mix of jackfruit leaves and Cassia flowers had higher phenolic content and antioxidant activity than JLT.

\subsection{Effect of herbal teas on glycemic response}

Sixteen healthy subjects were recruited in the study and there was one drop out from the study due to change of personal work arrangement. The mean age of the 15 remaining subjects, 11 males and 4 females, was $24 \pm 2$ y and their mean body mass index was $21.7 \pm 3.4 \mathrm{~kg} / \mathrm{m}^{2}$. The $24 \mathrm{~h}$ dietary recalls of the subjects revealed a daily intake of $1,322 \pm 257 \mathrm{kCal}$, from $216 \pm 49,39 \pm 8$, and $36 \pm$ $9 \mathrm{~g}$ of carbohydrate, protein and fat, respectively. None of subjects had any adverse effects due to the ingestion of herbal teas during the study period.

Table 5 and Figure 1 present the change of mean PGC of healthy adults over the time from baseline $(0 \mathrm{~min})$ to $120 \mathrm{~min}$. All tested herbal teas showed lower PGC than the control of glucose solution at each postprandial time point except at the baseline (Figure 1a). However only two herbal teas, namely JLT and JCT showed significantly lower $(p<0.05)$ PGC compared with that of the control at different postprandial times (Figure $1 \mathrm{~b}$ and Table 5). The reduction of PGC at time points of 30, 45, $60 \mathrm{~min}$ by JLT and JCT were significantly lower $(p<0.05)$ than the control and demonstrated the highest hypoglycemic efficacies at $60 \mathrm{~min}$ after ingestion of herbal teas. JCT is a blend of Cassia flowers and buds and Jackfruit leaves. The TPC and TEAC of JCT was lower than that of CFT, however, the results showed that the reduction of PGC did not follow this trend. Instead, considering the results obtained at $60 \mathrm{~min}$, JCT showed a higher reduction of PGC $(10.1 \%)$ than that of CFT.

Therefore, a slight but significant positive synergy could be ex- plained from these unexpected results. In presence of mixture of bioactive compounds those acting on intracellular targets can exert synergistic effects. Hellmann et al (2010) showed a synergetic effect of epigallocatechin gallate and digitonin on plasmodium sporozoite survival and motility.

There are limited human studies investigating how JLT, and CFT improve the postprandial glycemic control and none have reported the efficacy of JCT. This study showed that ingestion of a single dose of $250 \mathrm{~mL}$ of JCT herbal tea with $50 \mathrm{~g}$ of glucose challenge improved postprandial glycemic response in healthy adults.

Previous study showed an improvement of the glucose tolerance in normal individuals and maturity on set diabetic patients upon the ingestion of aqueous extract of $A$. heterophyllus leaves (Fernando et al., 1991). Fernando et al. (1990) demonstrated improved glucose tolerance in Sprague-Dawley rats upon the administration of aqueous A. heterophyllus extracts. Chandrika et al. (2006) showed that administration of flavonoid fraction from hot water extract of jackfruit leaves to normal Wistar rats lowered postprandial blood glucose concentration compared to those of the control. The significant reductions compared to the control were reported at $60 \mathrm{~min}(17.5 \%)$ and $120 \mathrm{~min}(37 \%)$ after administration. Their results revealed that flavonoid fraction was effective in reducing postprandial blood glucose level (Chandrika et al., 2006). Furthermore, a streptozotocin (STZ) diabetic induced animal model showed a postprandial hypoglycemic effect upon administration of ethyl acetate fraction of jackfruit leaf constituents (Chackrewarthy et al., 2010).

In the present study the maximum reduction of JLT and JCT were reported at $60 \mathrm{~min}$ and compared to the control showed 29 and $32 \%$ reduction, respectively (Table 5 ). The possible reason for lower PGC could be the lower intestinal absorption of glucose as concentrations were measured within the time course of 120 min. The high content of epigallocatechin derivative present in Jackfruit leaves could be responsible for the reduction of PGC in the present work by JLT and JCT. Kobayashi et al. (2000) showed that dietary glucose uptake can be inhibited by tea polyphenols such as epicatechin gallate and epigallocatechin gallate which interact with sodium-dependent glucose transporter, SGLT1. The transport activity of SGLT1 was inhibited in a competitive manner by epicatechin gallate controlling glucose absorption at the intestinal tract (Shimizu et al., 2000). In addition, multiple dose studies of jackfruit leaf extract on rat models showed the reduction of fasting blood glucose levels and improved other health

Table 5. Difference of postprandial plasma glucose response of healthy adults on herbal teas compared to the control

\begin{tabular}{|c|c|c|c|c|c|c|}
\hline & $0 \mathrm{~min}$ & $30 \mathrm{~min}$ & $45 \mathrm{~min}$ & $60 \mathrm{~min}$ & $90 \mathrm{~min}$ & $120 \mathrm{~min}$ \\
\hline Cassia flowers tea (CFT) & $-4.9(3.8)$ & $10.3(7.2)$ & $7.8(7.9)$ & $18.5(7.3)$ & $8.2(7.4)$ & $4.9(5.1)$ \\
\hline Jackfruit leaves tea (JLT) & $-4.9(4.2)$ & $20.0 *(9.4)$ & $28.1^{*}(7.8)$ & $28.8 *(5.5)$ & $23.2 *(3.8)$ & $3.9(3.2)$ \\
\hline Jackfruit leaves + Cassia flowers tea (JCT) & $-8.5(3.6)$ & $20.1 *(6.7)$ & $29.6 *(9.1)$ & $31.7 *(8.5)$ & $16.2(5.8)$ & $2.4(5.0)$ \\
\hline
\end{tabular}

Values are mean difference (SEM); $\mathrm{n}=14 ;{ }^{*}$ Difference from control is significant $(p<0.05)$. 

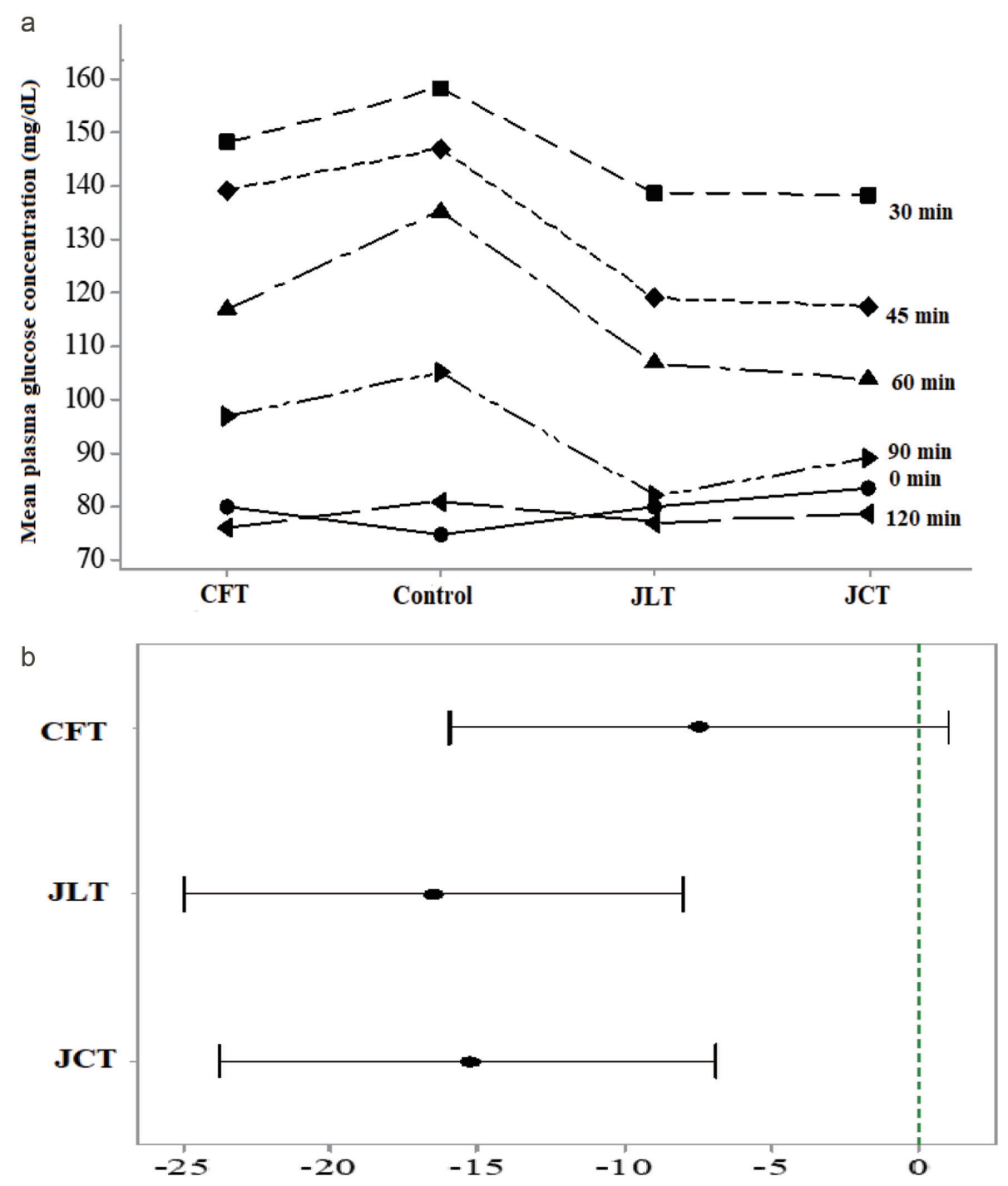

Figure 1. (a) Mean plasma glucose concentration of healthy adults (herbal tea $\times$ time); (b) Comparison of glycemic response of test herbal teas with the control (If an interval does not contain zero, the corresponding mean is significantly different from the control mean). CFT, Cassia flowers tea; CJT, Cassia flowers and Jackfruit leaves mix tea; JLT, Jackfruit leaves tea.

outcomes, suggesting beneficial effects of JLT and JCT dietary supplementation in the long run (Omar et al., 2011; El-Azim et al., 2015). Omar et al. (2011) showed that administration of ethanol or n-butanol extracts of jackfruit leaves for 10 days reduced fasting blood glucose level of STZ induced diabetic rats by 72 and $61 \%$, respectively, compared to the untreated diabetic rats. ElAzim et al. (2015) reported that administration of aqueous extract of jackfruit leaves to diabetic rats for 8 weeks partially reversed the elevated fasting blood glucose levels. These improved effects could be due to epigallocatechin derivative and other flavonoids found in aqueous extract of jackfruit leaves. Previous studies have shown that dietary supplementation of tea epigallocatechin gallate (EGCG) in multiple doses improved glucose tolerance and increased glucose stimulated insulin secretion in animal models (Wolfram et al., 2006; Ortsäter et al., 2012). In another study reduction of hepatic glucose production by EGCG was demonstrated (Waltner-Law et al., 2002). Furthermore, Kotowaroo et al. (2006) showed $\alpha$-amylase inhibition in vitro by the aqueous ex- tract of the jackfruit leaves.

\subsection{Effect of herbal teas on plasma antioxidant capacity (PAC)}

The PAC of healthy subjects were measured at the baseline and at $120 \mathrm{~min}$ after ingestion of herbal teas. All herbal teas, namely CFT, JLT and JCT, showed a significant $(p<0.05)$ increment of PAC compared to the baseline and the change varied from 37.7 to $71.1 \%$. Furthermore, PAC of subjects after $120 \mathrm{~min}$ of ingestion of herbal teas were significantly higher than that of the control (Table 6). The increment of PAC for each herbal tea compared to the corresponding baseline varied from 87.8 to $134.5 \mu \mathrm{mol} \mathrm{TE} / \mathrm{L}$. Villano et al. (2010) demonstrated an increment of PAC in healthy adults after load of $500 \mathrm{~mL}$ single dose of fermented or unfermented rooibos teas. Furthermore, the increment of PAC for green tea beverage and green tea extract in adults with metabolic syndrome was reported by Basu et al. (2013). 
Table 6. Plasma antioxidant capacity ( $\mu \mathrm{mol}$ trolox equiv/L) of healthy adults at baseline and 120 min after ingestion of herbal teas

\begin{tabular}{lllll}
\hline & Baseline & $\begin{array}{l}\text { End } \\
(\mathbf{1 2 0} \text { min after ingestion) }\end{array}$ & $\begin{array}{l}\text { Difference between } \\
\text { baseline and end }\end{array}$ & $\begin{array}{l}\text { Percentage change (\%) com- } \\
\text { pared to baseline at the end }\end{array}$ \\
\hline Control & $206.9 \pm 44.1^{\mathrm{a}}$ & $196.1 \pm 43.5^{\mathrm{a}}$ & -10.8 & - \\
Jackfruit leaves tea (JLT) & $237.7 \pm 26.2^{\mathrm{a}}$ & $325.5 \pm 54.2^{\mathrm{b}}$ & 87.8 & $37.7^{*}$ \\
Cassia flowers tea (CFT) & $207.7 \pm 28.4^{\mathrm{a}}$ & $342.2 \pm 36.0^{\mathrm{b}}$ & 134.5 & $67.0^{*}$ \\
Jackfruit leaves + Cassia flowers tea (JCT) & $204.8 \pm 38.4^{\mathrm{a}}$ & $338.9 \pm 64.4^{\mathrm{b}}$ & 134.1 & $71.1^{*}$ \\
\hline
\end{tabular}

All values are mean $\pm \mathrm{SEM} ; \mathrm{n}=14$; Values in each column with the same letter are not significantly different $(p>0.05)$; Values in each row having the same letter are not significantly different $(p>0.05)$. *Percentage increment of plasma antioxidant capacity of each tea compared to the respective baseline is significant $(p>0.05)$.

The CFT showed a significantly higher difference of PAC of $\mu \mathrm{mol} 134.5 \mathrm{TE} / \mathrm{L}$ between baseline and at the end of $120 \mathrm{~min}$ after ingestion compared to other herbal teas. Furthermore, CFT and JCT showed high increment of PAC of 67.0 and $71.1 \%$, respectively, at the end of 120 min compared to the baseline value. JLT prepared with jack fruit leaves alone showed only $37.7 \%$ increment of PAC. In the present work the compositional analysis showed that CFT contained a number of compounds belonging to several phenolic groups. Only ellagic acid and epigallocatechin derivatives were identified in JLT (Table 1). In a previous study Gurib-Fakim et al., (1995) reported the content of cycloartenone, cycloartenol, $\beta$ sitesterol and tannin in the leaves. All these phenolic compounds are potent antioxidants and may render beneficial effects to prevent and manage non-communicable disease conditions. It is noteworthy that JCT which is a mixture of Jackfruit leaves and Cassia showed the highest increment of PAC suggesting a synergistic effect of ingredients on the plasma antioxidant activity.

The foregoing results show that selected herbal teas are potential sources of antioxidant compounds and are effective in attenuating oxidative stress that leads to a plethora of non-communicable diseases, including type 2 diabetes. It is also suggested that these teas have the potential to reduce hyperglycemic conditions. However, in the present work hypoglycemic efficacy was determined against a pure glucose challenge in healthy adults who can adjust blood glucose concentration under normal carbohydrate metabolism. Hence, further studies are in progress to understand the efficacy of herbal teas against different carbohydrate loads as a meal in healthy and diabetic individuals. Furthermore, investigation of long-term efficacy of herbal teas with multiple doses in healthy and hyperglycemic individuals is underway.

\section{Conclusion}

Herbal teas of Cassia flowers and buds, and jackfruit leaves of the firm variety are rich sources of water-soluble phenolic compounds which demonstrated potent antioxidant activity. All tested herbal teas increased the plasma antioxidant capacity, thus demonstrating the potential of herbal teas in reducing oxidative stress. Hypoglycemic efficacy also varied with the type of herbal tea considered. Although Cassia flower herbal teas are rich sources of phenolic compounds such as flavonoids, jackfruit leaves and mix of jackfruit leaves and Cassia flowers herbal teas proved to be most effective hypoglycemic dietary supplement suggesting different effects of the constituents present in herbal teas.

\section{Acknowledgments}

This research was supported by the Wayamba University of Sri
Lanka through a University research grant (SRHDC/RP/04/18-03) to AC. Authors acknowledge Thavanthen Januka, Disna Kumari of the Department of Applied Nutrition for the assistance in the study, Dr Gamilka Prathapasinghe, Department of Livestock and Avian Sciences of Faculty of Livestock, Fisheries and Nutrition for providing laboratory facilities.

\section{Conflict of interest}

The authors state that there is no conflict of interest to declare.

\section{References}

Abesundara, K.J., Matsui, T., and Matsumoto, K. (2004). Alpha-Glucosidase inhibitory activity of some Sri Lanka plant extracts, one of which, Cassia auriculata, exerts a strong antihyperglycemic effect in rats comparable to the therapeutic drug acarbose. J. Agric. Food Chem. 52(9): 2541-5.

Akalin, K., Ekiz, A.T., Karakaya, F., Karadag, A., Pelvan, E., Doğan, K., Alasalvar, C., and Aksu, S. (2019). In vivo antidiabetic activities of green and black tea polysaccharides using streptozotocin - induced diabetic mice fed with a high-fat diet. J. Food Bioact. 8: 74-83.

Andjelković, M., Van Camp, J., De Meulenaer, B., Depaemelaere, G., Socaciu, C., Verloo, M., and Verhe, R. (2006). Iron-chelation properties of phenolic acids bearing catechol and galloyl groups. Food Chem. 98(1): 23-31.

Basu, A., Betts, N.M., Mulugeta, A., Tong, C., Newman, E., and Lyons, T.J. (2013). Green tea supplementation increases glutathione and plasma antioxidant capacity in adults with the metabolic syndrome. Nutr. Res. 33(3): 180-187.

Biworo, A., Tanjung, E., Iskandar, Khairina, and Suhartono, E. (2015). Antidiabetic and antioxidant activity of jackfruit (Artocarpus heterophyllus extract. J. Med. Bioeng. 4(4): 318-323.

Caesar, L.K., and Cech, N.B. (2019). Synergy and antagonism in natural product extracts: when $1+1$ does not equal 2. Nat. Prod. Rep. 36(6): 869-888.

Chackrewarthy, S., Thabrew, M.I., Weerasuriya, M.K.B., and Jayasekera, S. (2010). Evaluation of the hypoglycemic and hypolipidemic effects of an ethylacetate fraction of Artocarpus heterophyllus (jak) leaves in streptozotocin-induced diabetic rats. Pharmacogn. Mag. 6(23): 186-190.

Chandrasekara, A., and Shahidi, F. (2018). Herbal Beverages: Bioactive compounds and their role in disease risk reduction - A Review. J. Trad. Comp. Med. 8(4): 451-458.

Chandrasekara, A., and Shahidi, F. (2010). Content of insoluble bound phenolics in millets and their contribution to antioxidant capacity. J. Agric. Food Chem. 58(11): 6706-6714.

Chandrasekara, A., Daugelaite, J., and Shahidi, F. (2018). DNA scission and LDL cholesterol oxidation inhibition and antioxidant activities of bael (Aegle marmelos) flower extracts. J. Trad. Comp. Med. 8(3): 428-435.

Chandrasekara, A., Januka, T., Kumari, D., de Camargo, A.C., and Shahidi, F. (2020). Phenolic antioxidants of bael fruit herbal tea and effects 
on postprandial glycemia and plasma antioxidant status in healthy adults. J. Food Bioact. 11: 75-83.

Chandrika, U.G., Wedage, W.S., Wickramasinghe, S.M.D.N., and Fernando, W.S. (2006). Hypoglycaemic action of the flavonoid fraction of Artocarpus heterophyllus leaf. Afr. J. Trad. CAM 3(2): 42-50.

de Camargo, A.C., Biasoto, A.C.T., Schwember, A.R., Granato, D., Rasera, G.B., Franchin, M., Rosalen, P.L., Alencar, S.M., and Shahidi, F. (2019). Should we ban total phenolics and antioxidant screening methods? The link between antioxidant potential and activation of NF-KB using phenolic compounds from grape by-products. Food Chem. 30(290): 229-238.

de Camargo, A.C., Regitano-d'Arce, M.A.B., Gallo, C.R., and Shahidi, F. (2015). Gamma-irradiation induced changes in microbiological status, phenolic profile and antioxidant activity of peanut skin. J. Funct. Foods. 12: 129-143.

de Camargo, A.C., and Schwember, A.R. (2019). Phenolic-driven sensory changes in functional foods. J. Food Bioact. 5: 6-7.

El-Azim, S.A.A., El- Raheem, M.T.A., Said, M.M., and Abdeen, M.A. (2015). Effects of mulberry and jackfruit leaf extracts on blood glucose, oxidative stress and DNA damage in STZ/NA-induced diabetic rats. Diabetologia Croatica 44(1): 3-19.

Fernando, M.R., Thabrew, M.I., and Karunanayake, E.H. (1990). Hypoglycaemic activity of some medicinal plants in Sri Lanka. Gen. Pharmacol. 21(5): 779-782.

Fernando, M.R., Wickremasinghe, S.M., Thabrew, M.I., Ariyananda, P., and Karunanayake, E.H. (1991). Effect of Artocarpus heterophyllus and Asteracanthus longifolia on glucose tolerance in normal human subjects and in maturity onset diabetic patients. J. Ethanopharmacol. 31: 277-82.

Gurib-Fakim, A., Gueho, J., and Bissoondoyal, M.D. (1995). Plantes Medicinales de Maurice. Tome 1. Editions de I'Ocean Indien, Rose Hill, Mauritius, pp. 1-492.

Hellmann, J.K., Munter, S., Wink, M., and Frischknecht, F. (2010). Synergistic and additive effects of epigallocatechin gallate and digitonin on Plasmodium sporozoite survival and motility. Plos One 5(1): e8682.

Iannitti, T., and Palmieri, B. (2009). Antioxidant therapy effectiveness: an up to date. Eur. Rev. Med. Pharmacol. Sci. 13(4): 245-278.

International Diabetes Federation. (2019). Diabetes Atlas, 9th edn. Brussels, Belgium. Available at: https://www.diabetesatlas.org.

Juan-Badaturugae, M., Habtemariam, S., and Thomas, M.J.K. (2011). Antioxidant compounds from a South Asian beverage and medicinal plant, Cassia auriculata. Food Chem. 125(1): 221-225.

Kalaivani, A., and Umamaheswari, A. (2008). Anti-hyperglycemic and antioxidant properties of Cassia auriculata leaves and flowers on alloxan induced diabetic rats. Pharmacol. 1: 204-217.

Kandil, F.E., Grace, M.H., Seigler, D.S., and Cheeseman, J.M. (2004). Polyphenolics in Rhizophora mangle L. leaves and their changes during leaf development and senescence. Trees 18: 518-528.

Kobayashi, Y., Suzuki, M., Satsu, H., Arai, S., Hara, Y., Suzuki, K., Miyamoto, Y., and Shimizu, M. (2000). Green tea polyphenols inhibit the sodiumdependent glucose transporter of intestinal epithelial cells by a competitive mechanism. J. Agric. Food Chem. 48(11): 5618-5623.

Kotowaroo, M.I., Mahomoodally, M.F., Gurib-Fakim, A., and Subratty, A.H. (2006). Screening of traditional antidiabetic medicinal plants of Mauritius for possible alpha-amylase inhibitory effects in vitro. Phytother. Res. 20: 228-31.

Kumari, D., Chandrasekara, A., and Shahidi, F. (2019). Bioaccessibility and antioxidant activities of finger millet food phenolics. J. Food Bioact. 6: 100-109.

Kumari, D., Chandrasekara, A., Athukorale, P., and Shahidi, F. (2020). Finger millet porridges subjected to different processing conditions showed low glycemic index and variable efficacy on plasma antioxidant capacity of healthy adults. Food Prod. Proces. Nut. 2(13): 1-11.

Lee, W.Y., Emmy, H.K.I., Abbe Maleyki, M.J., and Amin, I. (2007). Antioxidant capacity and phenolic content of selected commercially available cruciferous vegetables. Malaysian J. Nutr. 13(1): 71-80.

Li, L., and Tsao, R. (2019). UF-LC-DAD-MS ${ }^{n}$ for discovering enzyme inhibitors for nutraceuticals and functional foods. J. Food Bioact. 7: 27-35.

Li, S., Gosslau, A., Lange, K., and Ho, C.-T. (2019). Profiled tea extracts exemplifying the importance of characterizing food bioactives: opinion piece. J. Food Bioact. 5: 1-5.
Loizzo, M.R., Tundis, R., Chandrika, U.G., Abeysekera, A.M., Menichini, F., and Frega, N.G. (2010). Antioxidant and antibacterial activities on foodborne pathogens of Artocarpus heterophyllus Lam. (Moraceae) leaves extracts. J. Food Sci. 75(5): 291M-295M.

Milton, F. (1937). The use of ranks to avoid the assumption of normality implicit in the analysis of varian. J. Am. Stat. Assoc. 32(200): 675-701.

Omar, H.S., El-Beshbishy, H.A., Moussa, Z., Taha, K.F., and Singab, A.N.B. (2011). Antioxidant activity of Artocarpus heterophyllus Lam. (jack fruit) leaf extracts: remarkable attenuations of hyperglycemia and hyperlipidemia in streptozotocin-diabetic rats. Sci. World J. 11: 788-800.

Ortsäter, H., Grankvist, N., Wolfram, S., Kuehn, N., and Sjöholm, A. (2012) Diet supplementation with green tea extract epigallocatechin gallate prevents progression to glucose intolerance in $\mathrm{db} / \mathrm{db}$ mice. Nutr. Metab. (Lond). 14(9): 11.

Pinaffi, A.C.C., Sampaio, G.R., Soares, M.J., Shahidi, F., de Camargo, A.C., and Torres, E.A.F.S. (2020). Insoluble-Bound Polyphenols Released from Guarana Powder: Inhibition of Alpha-Glucosidase and Proanthocyanidin Profile. Molecules 25: 679.

Prakash, O., Kumar, R., Mishra, A., and Gupta, R. (2009). Artocarpus heterophyllus (Jackfruit): An Overview. Phcog Rev. 3(6): 353-358.

Rama Rao, A.V., Varadan, M., and Venkataraman. (1973). Colouring matter of the A. hetetophyllus. Indian J. Chem. 11: 298-299.

Re, R., Pellegrini, N., Proteggente, A., Pannala, A., Yang, M., and RiceEvans, C. (1999). Antioxidant activity applying an improved ABTS radical cation decolorization assay. Free Rad. Biol. Med. 26(9-10): 1231-1237.

Shahidi, F., Ramakrishnan, V., and Oh, W.Y. (2019). Bioavailability and metabolism of food bioactives and their health effects: a review. J. Food Bioact. 8: 6-41.

Shimizu, M., Kobayashi, Y., Suzuki, M., Satsu, H., and Miyamoto, Y. (2000). Regulation of intestinal glucose transport by tea catechins. Biofactors. 13(1-4): 61-65.

Silva, C.P., Soares-Freitas, R.A.M., Sampaio, G.R., de Camargo, A.C., and Torres, E.A.F.S. (2019). Guarana as a source of bioactive compounds. J. Food Bioact. 6: 1-5.

Singleton, V.L., and Rossi, J.A. (1965). Colorimetry of total phenolics with phosphomolybdic-phosphotungstic acid reagents. Am. J. Enol Vitic. 16: $144-158$.

Swami, S.B., Thakor, N.J., Haldankar, P.M., and Kalse, S.B. (2012). Jackfruit and its many functional components as related to human health: a review. Comp. Rev. Food Sci. Food Safety 11(6): 565-576.

Thapa, N., Thapa, P., Bhandari, J., Niraula, P., Shrestha, N., and Shrestha, B.G. (2016). Study of phytochemical, antioxidant and antimicrobial activity of Artocarpus heterophyllus. Nepal J. Biotech. 4(1): 47-53.

Tipduangta, P., Julsrigival, J., Chaithatwatthana, K., Pongterdsak, N., Tipdu angta, P., and Chansakaow, S. (2019). Antioxidant Properties of Thai Traditional Herbal Teas. Beverages 5(3): 44.

Villano, D., Pecorari, M., Testa, M.F., Raguzzini, A., Stalmach, A., Crozier, A., Tubili, C., and Serafini, M. (2010). Unfermented and fermented rooibos tea (Aspalathus linearis) increase plasma total antioxidant capacity in healthy humans. Food Chem. 123(3): 679-683.

Waltner-Law, M.E., Wang, X.L., Law, B.K., Hall, R.K., Nawano, M., and Granner, D.K. (2002). Epigallocatechin gallate, a constituent of green tea, represses hepatic glucose production. J. Biol. Chem. 277(38): 3493334940.

Wang, X.L., Di, X.X., Shen, T., Wang, S.Q., and Wang, X.N. (2017). New Phenolic compounds from the leaves of Artocarpus heterophyllus. Chinese Chem. Let. 28: 37-40.

Wolfram, S., Raederstorff, D., Preller, M., Wang, Y., Teixeira, S.R., Riegger, C., and Weber, P. (2006). Epigallocatechin gallate supplementation alleviates diabetes in rodents. Nutr. 136(10): 2512-2518.

Yeo, J., and Shahidi, F. (2019). Revisiting DPPH (2,2-diphenyl-1-picrylhydrazyl) assay as a useful tool in antioxidant evaluation: A new IC100 concept to address its limitations. J. Food Bioact. 7: 36-42.

Zhang, H., and Tsao, R. (2016). Dietary polyphenols, oxidative stress and antioxidant and anti-inflammatory effects. Curr. Opinion Food Sci. 8 : 33-42.

Zhao, C.-N., Tang, G.-Y., Cao, S.-Y., Xu, X.-Y., Gan, R.-Y., Liu, Q., Mao, Q.-Q., Shang, A., and Li, H.-B. (2019). Phenolic profiles and antioxidant activities of 30 tea infusions from green, black, Oolong, white, yellow and dark teas. Antioxidants 8(7): 215. 